\title{
Obstruction of the third part of the duodenum in tuberculosis
}

\author{
A.K. Kriplani*, Sunil Kumar and L.K. Sharma \\ Department of Surgery, All India Institute of Medical Sciences, Ansari Nagar, New Delhi 110 029, India.
}

\begin{abstract}
Summary: Extrinsic compression by tuberculous mesenteric nodes is the rarest form of duodenal obstruction and one such case is presented. Radiology is helpful in differentiating various duodenal lesions. Ideal surgical treatment is duodeno-jejunostomy but in some cases only gastroenterostomy is possible.
\end{abstract}

\section{Introduction}

Isolated obstruction of the third part of the duodenum is rare. Causes of infrapapillary duodenal obstruction include midgut volvulus with transduodenal band, duodeno-mesocolic band, arterio-mesenteric ileus, tumours of the pancreas, duodenal neoplasia, traumatic or inflammatory strictures and pressure by lymph nodes. Duodenal obstruction due to extrinsic pressure by tuberculous mesenteric glands is very rare and one such case is presented.

\section{Case report}

A 45 year old male presented with fullness of the upper abdomen after meals for 6 months and bilious vomiting for last 2 months. Vomiting relieved him of the discomfort. He had lost $5 \mathrm{~kg}$ in weight in the last 6 months. There was no history of melaena or fever. Abdominal examination revealed epigastric tenderness, visible gastric peristalsis and sucussion splash 2 hours after meals. There was no lymphadenopathy.

Laboratory findings were all within normal range except mild anaemia (haemoglobin $10.2 \mathrm{~g} / \mathrm{dl}$ ) and elevated erythrocyte sedimentation rate $(80 \mathrm{~mm}$ at one hour). Chest radiogram showed evidence of tuberculosis in the right apical region. Barium meal examination revealed dilatation of first and second parts of the duodenum and delayed transit of the contrast through the transverse part of the duodenum. The smooth outline of the obstructed segment was suggestive of pressure by an extrinsic mass.

On gastroduodenoscopy no abnormality could be seen in the duodenal mucosa but the instrument could not be passed beyond the obstruction. Multiple biop-

*Correspondence and present address: A.K. Kriplani, M.B. B.S., M.S., J.L.N. Medical College, Ajmer, Rajasthan 305001 , India.

Accepted: 8 April 1986 sies from the duodenal mucosa revealed no abnormality.

At laparotomy the entire parietal and visceral peritoneum was covered with multiple tubercles of 2 to $5 \mathrm{~mm}$ in size which were more numerous over the duodenum. The stomach and proximal duodenum were dilated. A large mass of nodes at the upper part of the mesentery was compressing the transverse part of the duodenum. Duodeno-jejunostomy was considered inadvisable due to the presence of bulky nodes and the friable serosa of the duodenum. A posterior, retrocolic gastroenterostomy was performed and truncal vagotomy was added to protect the jejunal mucosa from gastric acidity. Biopsies were taken from omentum and lymph nodes which subsequently confirmed the suspicion of tuberculosis. Antitubercular treatment was started from the first postoperative day. Recovery was uneventful.

\section{Discussion}

Although intestinal tuberculosis is not an uncommon disease in many parts of the world, duodenal obstruction in tuberculosis is extremely unusual. More often duodenal involvement in tuberculosis leads to formation of stricture and Lockwood et al. (1974) collected 144 such cases. A less common form of obstruction is pressure by enlarged tuberculous nodes at the root of the mesentery. Mallory (1942) reported a case of duodenal obstruction by enlarged nodes which on autopsy proved to be tubercular. Davey \& Pearson (1965) gave an account of 5 cases with duodenal obstruction in the transverse part, two of them by tuberculous nodes. Chawla \& Berry (1969) described 4 cases of duodenal obstruction due to tuberculous nodes. Despite an extensive search, we could not find any report after 1969. This perhaps represents effectiveness and availability of chemotherapy since most 
cases of duodenal tuberculosis occur only with widespread disease.

While tubercular strictures can occur anywhere in the duodenum and may even be multiple, mesenteric adenopathy compresses the transverse part of the duodenum. On barium meal study the obstruction can be seen either as a sharp cutoff or a smooth tapering beak (Davey \& Pearson, 1965). Gastroduodenoscopy may be required to rule out mucosal involvement.

Differentiation from other causes of extrinsic pressure may not be easy, particularly arterio-mesenteric ileus, which is more common. Characteristic radiological signs include a sharp, linear, oblique line which points towards the right iliac fossa (Hartenstein et al., 1961) with relief of obstruction in the prone position and its accentuation in the upright position on cinefluorography (Anderson et al., 1973). The tuberculous nature of the pathology may be suspected if there is past history or concomitant evidence of

\section{References}

ANDERSON, W.C., VIVIT, R., KIRSCH, I.E. \& GREENLEE, H.B. (1973). Arterio-mesenteric duodenal compression syndrome; its association with peptic ulcer. American Journal of Surgery, 125, 681.

CHAWLA, S. \& BERRY, K. (1969). Duodenal obstruction due to tubercular lymphadenitis. A study of four cases. Clinical Radiology, 20, 99.

DAVEY, W.W. \& PEARSON, J.B. (1965). Obstruction of the third part of the duodenum. British Journal of Surgery, 52, 189. tuberculosis in any other organ. Abnormalities on chest X-ray are present in $50 \%$ of patients with duodenal tuberculosis.

Surgical correction may be required on an emergency basis if vomiting is persistent and severe. The ileocaecal region should be examined thoroughly since another obstruction may be present in this location. Duodenojejunostomy is ideal but may not always be possible due to caseating nodes in the vicinity (Davey \& Pearson, 1965) and gastro-enterostomy may be the only alternative available. Post-operative chemotherapy is always required and if the obstruction is not severe and the patient's general condition is satisfactory, reliance on chemotherapy alone is advocated (Davey \& Pearson, 1965).

In countries where tuberculosis is still prevalent, it should be remembered as one of the causes of duodenal obstruction.

HARTENSTEIN, P.E., ZIPERMAN, H.H. \& SMITH, M.L. (1961). Infra-papillary obstruction of the duodenum. Annals of Surgery, 154, 125.

LOCKWOOD, C.M., FORSTER, P.M., CATTO, J.V.F. \& STEWART, J.S. (1974). A case of duodenal tuberculosis. American Journal of Digestive Diseases, 19, 575.

MALLORY, T.B. (1942). Case records of the Massachusetts General Hospital: Case 28192. New England Journal of Medicine, 226, 773. 